\title{
Research on Destriping Based on Rough Set
}

\author{
Xiangyang Chen, Liping Chen, Sile Wang, Wenzhu Yang, Sukui Lu \\ School of Computer Science and Technology, Hebei University, Baoding, China \\ Email: againcome@sina.com
}

Received August 2015

\begin{abstract}
In order to remove the stripe noises in cotton foreign fiber images by line scanning camera collected, in multi threshold segmentation of rough set, every region's color is instead of the statistics color of the region. This method can retain the detail information of original image as far as possible, and do well in the stripe noise removal. The roughness of rough set was calculated respectively using directional diagram, Canny operator and Sobel operator. Comparing the three methods, the results indicate that the Canny operator keeps the more details of image, and directional diagram and Sobel operator have the better effects on denoising.
\end{abstract}

\section{Keywords}

Stripe Noises, Multi Threshold Segmentation, Rough Set, Denosing

\section{Introduction}

In using line scan camera to acquire the cotton fiber image, it is often to produce stripe noises, which affects the extraction and recognition of the foreign fiber [1]. Today there are many methods to remove the stripe noises. The traditional method of denoising can be divided into the frequency domain and the spatial domain. Frequency domain denoising depends that the noise frequency is different larger with the image frequency, and therefore with the noise of the image is converted from the spatial domain to the frequency domain, to change the transforming coefficient of the noise, as far as possible to retain the transforming coefficients of the image information, and then inversing transform image to reducing image noises. But the denoising process tends to lose the part detail information of the image, resulting in blurred images. Commonly used methods are Wavelet transform [2] and Fourier transform [3]. Spatial denoising is mainly through calculating the image gray values, and using the normal pixel gray value as a reference to correct the noise pixel gray value, so as to reduce the influence of noise pixels. Typical methods are median filter [4], histogram equalization [5] [6] and the moment matching [7].

Using rough set theory [8] in multi threshold image segmentation has a good effect. If using statistical color replacement the color of segmentation region, it will realize the target image denoising. The distribution and intensity of stripe noise in the cotton fiber image is not uniform, in order to ensure the clarity of the image, the multi threshold segmentation of rough set is used to remove the noise.

\section{Rough Set Theory}

Since 1982 Poland scholar Z. Pawlak proposed the rough set theory, it has been a hot research topic in the field 
of artificial intelligence, and has been widely applied machine learning, data mining, process control and image processing fields.

Definition 1: information systems can be represented as a four tuple, the formalized definition is: $S=\{U, A, V, f\}$, in which $U$ : finite set of objects; $A$ : finite set off properties; $V: V=\bigcup_{p \in A} V_{p}, V_{p}$ is $p$ attribute domain; $f: U \times A \rightarrow V$ is general function, makes $f\left(x_{i}, q\right) \in V_{q}$ for each $x_{i} \in U, q \in A$.

Definition 2: $X \subset U$ is a subset of individuals on the global, $P \subseteq A$. The lower approximation and the upper approximation of the $X$ are respectively

$$
\begin{gathered}
\underline{P} X=\{Y \in U \mid P: Y \subseteq X\} \\
\bar{P} X=\{Y \in U \mid P: Y \cap X \neq \varnothing\}
\end{gathered}
$$
$X$.

$\underline{P} X$ is the confirmed largest defined set contained within the $X, \bar{P} X$ is the smallest defined set contained Therefore, the boundary region can't be clearly defined as:

$$
\operatorname{Bnd}_{p}(X)=\bar{P} X-\underline{P} X
$$

The larger the boundary area is, the more the set $X$ can't express. The uncertainty of the set $X$ can be expressed by the roughness of rough sets, the formula is as follows:

$$
\rho_{P}(X)=1-\frac{\underline{P} X}{\bar{P} X}
$$

The $\rho_{P}(x)$ is $[0,1]$, the greater the value is, the more uncertainty the set $X$ is.

\section{Rough Set Denoising Process}

The core idea of rough set denoising is that the color of a segmentation region can be replaced by a same color, so as to achieve the purpose of noise removal. Therefore, how to determine the region is a core problem. The linear scanning image of the cotton fiber is a color image, and the information contained in the RGB color image is often more than the gray image, and it is more consistent with the human visual sense. If only gray level histogram is used to distinguish different gray areas, the approximate relationship between pixels in color image cannot be reflected.

The histogram can be used to express the number of pixels in each gray level, so the basic histogram can be used as the lower approximation of the region. The histon histogram considered the color difference between the pixels in the neighborhood, and the uncertain information is described. So the histon histogram is used as the upper approximation.

Let $f$ for a RGB color image of $M \times N$, which is decomposed into $f_{r}, f_{g}$ and $f_{b}$ in $R, G, B$ three color channels. The basic histogram and histon histogram are defined respectively:

Definition 3: basic histogram

$$
h_{i}(g)=\sum_{m=1}^{M} \sum_{n=1}^{N} \sigma\left(I\left(m, n, f_{i}\right)-g\right) ; 0 \leq g \leq L-1, i=\{R, G, B\}
$$

The $\sigma$ is an impulse function; the value $L$ is 256 , representing the number of gray level of images; $f_{i}$ represents a gray image; $I\left(m, n, f_{i}\right)$ is the gray value of pixel $I(m, n)$.

Definition 4: histon histogram

$$
H_{i}(g)=\sum_{m=1}^{M} \sum_{n=1}^{N}(1+X(m, n)) \sigma\left(I\left(m, n, f_{i}\right)-g\right) ; 0 \leq g \leq L-1, i=\{R, G, B\}
$$

$X(m, n)$ is a matrix of $M \times N$, which indicates that the difference of the neighborhood pixel color, can be obtained by the following Equation (7):

$$
X(m, n)= \begin{cases}1, & D(m, n)<\exp \\ 0, & \text { other }\end{cases}
$$


where $D(m, n)$ represents the distance sum of between each pixel and the center pixel point in the neighborhood, and exp represents a threshold constant.

The process of removal of the stripe noise using rough sets is shown in Figure 1. Since the histogram is calculated by the gray scale, it is necessary to decompose the RGB image from $R, G$ and $B$ three channels respectively.

Roughness calculation: the calculating the basic image histogram and histon histogram, then get the roughness images by Equation (4).

Bands calculation: due to many bands in the original image, in order to remove the noise information and need to unite the less different band. Firstly, the position vector and the number $N$ of all the peaks in the image are obtained according to the roughness, Height threshold Th of the peaks can be calculate by Equation (8):

$$
T h=M p-\sqrt{\sum_{i=1}^{N}\left(p_{i}-M p\right)^{2} / N}
$$

Which $M p$ is mean of the maximum and minimum peak value, $P_{i}$ is the peak point. The experimental results show that the best of the width threshold Tw of the peak is 10. Then the peaks of less than Th are abandoned, and the width of the peaks of less than Tw is united.

The minimum value between two peaks is the valley value, the gray level between the two valleys is a band.

Band's colors calculation: a band using a replace gray color, the color of band $\left[k_{1}, k_{2}\right]$ can be calculated by Equation (9):

$$
\text { BandColor }=\sum_{i=k 1}^{k 2} i * \frac{h(i)}{S H}
$$

The $h(i)$ is the number of pixels in the gray level $i$ of the basic histogram, $S H$ is the sum of the number of pixels between $\left[k_{1}, k_{2}\right]$, can be calculate by the Equation (10):

$$
S H=\sum_{i=k 1}^{k 2} h(i)
$$

A new composed gray color can be used to represent the color of the band.

In the $R, G$ and $B$ channels, the band information and band colors are calculated respectively, the color of each band is replaced by the calculating the gray color, and then to synthesize a RGB color image.

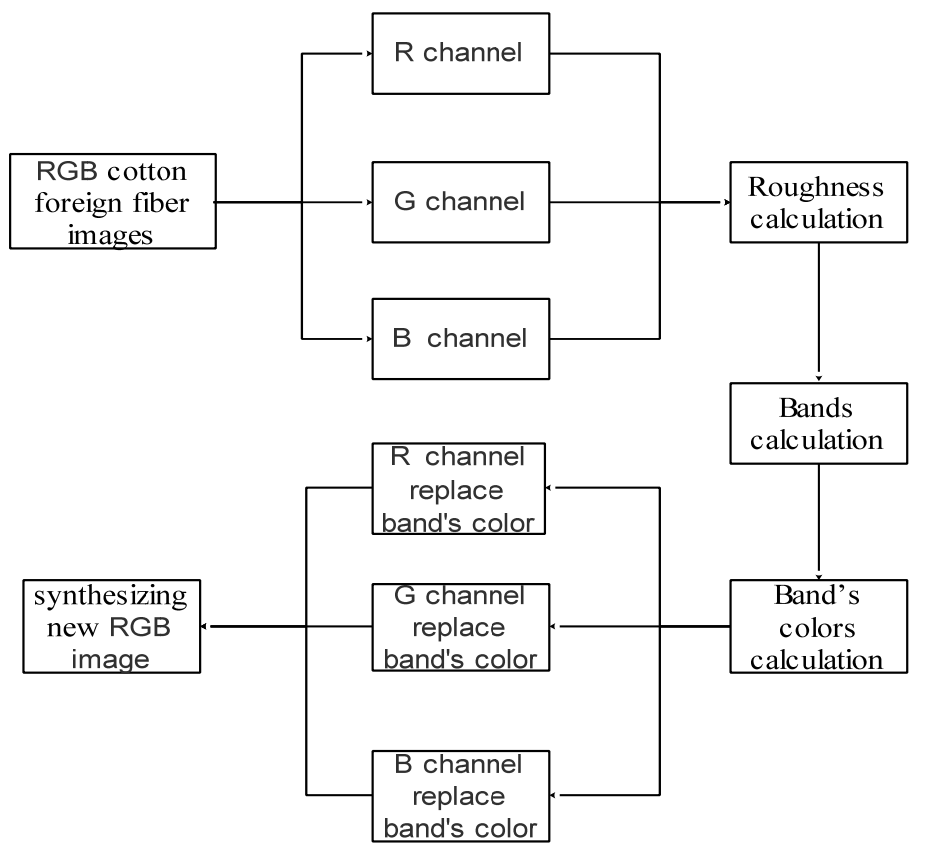

Figure 1. Rough set denoising process. 


\section{Experimental Results and Analysis}

Experimental picture is the image obtained from the color line scan camera. The key of rough set denosing is to determine the boundary of the color region, namely, the difference between the upper approximate histon his to gram $H(g)$ and the lower approximate basic histogram $h(g)$, so as to determine the roughness of the image. If the difference between the upper approximation and the lower approximation is obtained, that is the edge of the region, the roughness of the image can be calculated. Taking into account the image edge is the biggest difference between the images, the use of edge operator and the direction of the image should be able to get the approximate effect, so compared respectively with the three methods of denoising effect.

Method 1: directional diagram. $f(m, n)$ using the Equation (11) to calculate the distance between this point and $f(m-1, n-1)$ for the image $f$ of $M \times N$.

$$
\operatorname{ColorDis}_{(m, n)}=\operatorname{abs}(\arctan (f(m, n)-f(m-1, n-1)))
$$

and $1 \leq m \leq M, 1 \leq n \leq N$;

Method 2: using canny operator.

Method 3: using Sobel operator.

The experimental results are as follows:

Figure 2 is a cotton color image with a foreign fiber, among Figures 3-5, the (a) and (b) is the edge difference image and the denoising image by using the directional diagram, Canny operator and Sobel operator respectively. The experimental results show that the three methods do well in the strip noise removal. Compared with directional diagram and Sobel operator method, Canny operator method for denoising retains more image details, but some noise was also retained, so the denoising effects of the other two methods are better than its. These three methods can enhance the distinction between foreign fiber and others in the image. The rough set denosing not only realizes the removal of stripe noise, but also realizes the enhancement of the extraction region. It can provide higher quality image for the next step of image segmentation, and is helpful to the extraction and recognition of foreign fiber.

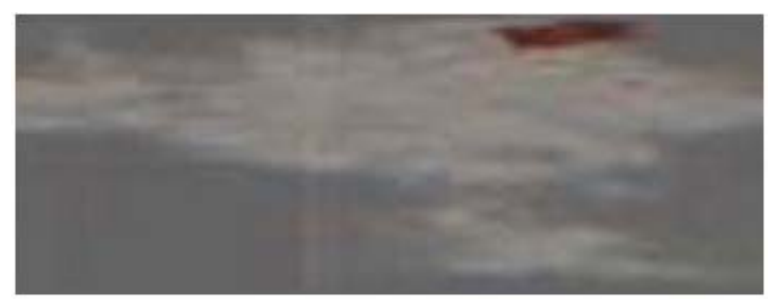

Figure 2. Color image of cotton.

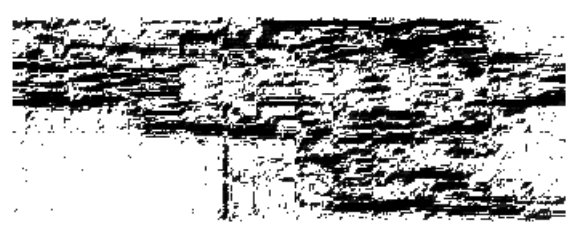

(a)

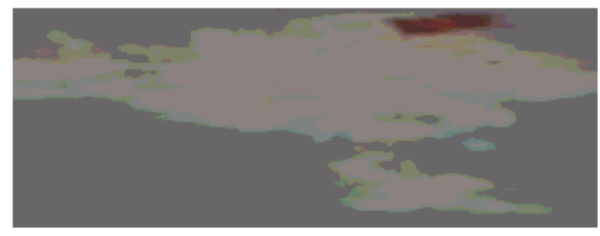

(b)

Figure 3. Directional diagram method.

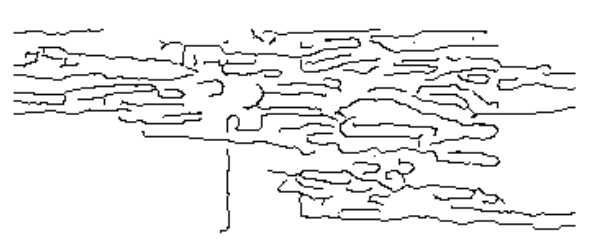

(a)

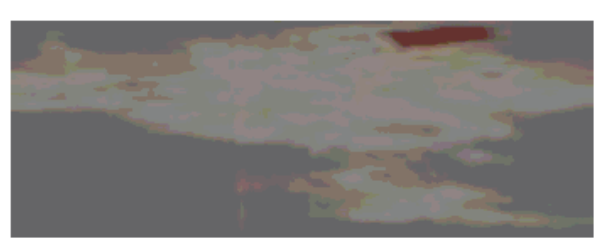

(b)

Figure 4. Canny operator method. 


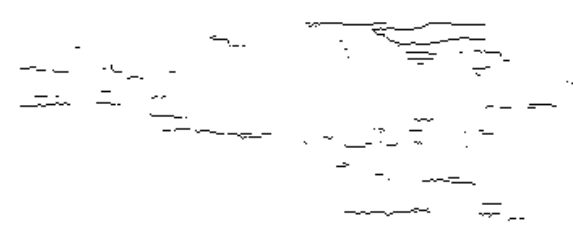

(a)

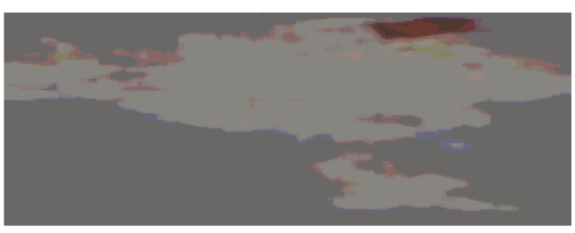

(b)

Figure 5. Sobel operator method.

\section{Conclusion}

The basic histogram and his ton histogram of the RGB color image is used as the lower approximation and the upper approximation of the image, and the roughness of the image is obtained by them. The image is divided into regions according to the roughness, and replaced the original color with statistical color within the region. The experimental results show that the rough set algorithm can achieve the removal of stripe noise very well. In the edge calculation of the image, the influences of the three methods are compared. Canny operator method has retained the more details, and the results of the direction and Sobel operator method are much better. The foreign fiber region is enhanced, which makes the foreground and background more obvious, and provides a high quality image for the following image segmentation.

\section{Acknowledgements}

The authors thank Hebei Natural Science Foundation (F2015201033), The Ministry of Science and Technology of the People's Republic of China (2013DFA11320), for their financial support.

\section{References}

[1] Wang, S.L., Fan, S.Y. and Lu, S.K. (2013) Visual Saliency Map Based Color Image Segmentation for Foreign Fiber Detection. Computer Engineering and Design, 34, 2783-2787.

[2] Lagha, M., Tikhemirine, M. and Bergheul, S. (2013) De-Noised Estimation of the Weather Doppler Spectrum by the Wavelet Method. Digital Signal Processing, 23, 322-328. http://dx.doi.org/10.1016/j.dsp.2012.08.001

[3] Houda, B., Rachid, S. and Ludovic, J. (2015) Comparison of Leaf Surface Roughness Analysis Methods by sensitivity to Noise Analysis. Biosystems Engineering, 136, 77-86. http://dx.doi.org/10.1016/j.biosystemseng.2015.04.012

[4] Huang, Y., Lei, T. and Fan, Y.Y. (2015) Adaptive Decision-Based Unsymmetric Trimmed Median Filter. Computer Science, 42, 303-307.

[5] Kang, C.Q., Zhang, Q.L. and Zheng, Y. (2013) Non Uniformity Correction Algorithm for IR Images Based on Midway Equalization Histogram. Laser \& Infrared, 43, 1240-1242.

[6] Anis, F.M.R., Hishammuddin, A. and Rohayanti, H. (2015) A Low Lighting or Contrast Ratio Visible Iris Recognition Using Iso-Contrast Limited Adaptive Histogram Equalization. Knowledge-Based Systems, 74, 40-48. http://dx.doi.org/10.1016/j.knosys.2014.11.002

[7] Jiang, W., Shen, H.F. and Zeng, Ch. (2014) Destriping Method for Band 28 of Terra MODIS Images. Geomatics and Information Science of Wuhan University, 39, 526-530.

[8] Wang, S., Zhu, Q.X. and Zhu, W. (2014) Graph and Matrix Approaches to Rough Sets through Matroids. Information Sciences, 288, 1-11. http://dx.doi.org/10.1016/j.ins.2014.07.023 